\title{
Les ONG et l'éducation dans les pays en développement
}

Introduction

Sandra Barlet et Jean-Pierre Jarousse

\section{OpenEdition}

\section{Journals}

Édition électronique

URL : http://journals.openedition.org/ries/2153

DOI : 10.4000/ries.2153

ISSN : 2261-4265

\section{Éditeur}

Centre international d'études pédagogiques

Édition imprimée

Date de publication : 1 décembre 2011

Pagination : 37-46

ISBN : 978-2-85420-593-0

ISSN : 1254-4590

\section{Référence électronique}

Sandra Barlet et Jean-Pierre Jarousse, "Les ONG et l'éducation dans les pays en développement », Revue internationale d'éducation de Sèvres [En ligne], 58 | décembre 2011, mis en ligne le 01 décembre 2011, consulté le 30 avril 2019. URL : http://journals.openedition.org/ries/2153 ; DOI : 10.4000/ ries. 2153 


\title{
Les ONG et l'éducation
}

\section{Introduction}

\section{Les ONG et l'éducation dans les pays en développement}

\author{
Sandra Barlet \\ Jean-Pierre Jarousse
}

Les organisations non gouvernementales (ONG) ou associations de solidarité, qu'elles agissent à l'échelle d'un pays ou à l'international, sont aujourd'hui, dans de nombreux domaines, les partenaires des États, des bailleurs de fonds ou financeurs et des populations, notamment celles qui ont le plus de difficultés à se faire entendre de la collectivité et de ses représentants. Elles sont très présentes dans le champ de l'éducation, sous des formes extrêmement variées. Le terme d'ONG, s'il peut être rapidement associé à quelques grandes structures et actions connues du grand public, renvoie en fait à des réalités très différentes en termes d'objectifs, de modes d'intervention, de gestion et d'organisation internes, mais aussi de financement, caractéristiques qui prennent en outre des colorations et des aspects différents selon les contextes géographiques, historiques, institutionnels, économiques et sociaux dans lesquels se situent les actions menées.

Pour simplifier, on peut a priori qualifier d'ONG toute forme d'association non publique, à but non lucratif, dont la vocation est d'apporter un service à des groupes identifiés ou de soutenir les actions à conduire en leur faveur.

La première caractéristique des ONG concerne leur statut «non gouvernemental » et donc leur autonomie par rapport au cadre d'action, aux modalités de financement et d'intervention de l'État. L'ONG bénéficie des avantages que procure le statut d'entreprise privée en termes de souplesse de gestion et d'organisation de ses activités tout autant que de désignation de ses objectifs et " clients" potentiels. Plus que l'État, qui a vocation à s'adresser à tous, elle peut décider de centrer ses activités sur des groupes particuliers, leur offrir des services qui ne sont pas ceux du lot commun, et diversifier en conséquence ses financements. Cette souplesse permet d'aborder des situations nouvelles ou peu traitées par la puissance publique, marginales, expérimentales. L'ONG peut de ce fait constituer un espace d'innovation et de créativité, offrir une réaction rapide à des situations urgentes, devenir le partenaire d'États relativement contraints par des objectifs et des cadres nationaux souvent rigides. Cette autonomie peut également permettre à l'ONG de prendre une certaine distance par rapport aux objectifs poursuivis par les États et/ou à leurs modes traditionnels d'intervention et de défendre des points de vue différents soit globalement, soit au bénéfice de groupes particuliers. 
La seconde caractéristique essentielle des ONG est leur statut d'organisme à but non lucratif. Cette caractéristique complète la précédente. L'ONG, au-delà du minimum exigé pour sa propre survie institutionnelle, obéit à des principes de fonctionnement et d'intervention altruistes qui la poussent à agir dans l'intérêt de groupes défavorisés, minoritaires, et pour promouvoir ces mêmes intérêts en tant que causes nationales et/ou internationales.

Au-delà de ces caractéristiques génériques, les ONG se différencient en termes d'activités, certaines privilégiant l'action directe, d'autres le " plaidoyer ${ }^{1}$ ». Les deux dimensions sont liées. La sphère géographique d'intervention de l'ONG est aussi un facteur de différentiation fort. Les positionnements et les types d'action des ONG diffèrent aussi selon les approches qu'elles adoptent, pour certaines fondées sur des publics spécifiques, pour d'autres sur des missions plus larges dans lesquelles s'inscrivent leurs interventions, à l'exemple du Gret, qui produit des références et des connaissances contribuant aux politiques publiques de développement et de coopération ${ }^{2}$.

Parce qu'elle relève de la dignité humaine et des droits de la personne mais aussi parce qu'elle cristallise de nombreuses inégalités sociales, l'éducation présente un enjeu qui justifie de nombreux « rappels » auprès des États, même lorsque ces derniers sont engagés par la signature de traités internationaux. Les actions de terrain vont donc largement de pair avec le rappel du «droit " à l'éducation et du plaidoyer pour une réduction significative des inégalités en termes d'accès à l'école ou de carrière scolaire. C'est souvent au nom de ces principes qu'agissent les ONG et, dans certains cas, leur action se concentre sur la promotion de ces principes.

\section{MISSIONS DES ONG EN MATIÈRE D’ÉDUCATION}

Dans le domaine de l'éducation, la place des ONG en tant que partenaires des États et des bailleurs multi- et bilatéraux a évolué avec l'histoire de l'aide internationale, et plus particulièrement avec les changements profonds survenus depuis le Forum mondial sur l'éducation qui s'est tenu à Dakar en 2000.

La conception la plus ancienne de l'aide dans le domaine de l'éducation, dans le mouvement des «ajustements structurels» et de la promotion de la privatisation des activités, accorde une confiance limitée aux institutions publiques nationales des pays en développement. Elle favorise une extériorisation des modalités de gestion et de mise en œuvre de l'aide, qui accorde une place

1. L'expression « plaidoyer international », désigne « l'ensemble des actions (campagnes, pétitions, organisations de contre-sommets...) conduites par des organisations issues de la société civile s'efforçant, à travers des activités de lobbying, d'influencer les décideurs politiques pour leur faire adopter des positions ou des textes favorables aux intérêts des pays en développement ou de leurs populations. " Joseph Zimet, Les ONG. De nouveaux acteurs pour changer le monde, Éditions Autrement/Paris, 2006, (Monde d'aujourd'hui), p. 73. (NdIR).

2. Le Gret est une association de solidarité internationale créée en 1976, qui regroupe sept cents professionnels du développement solidaire. Elle soutient des processus de développement durable dans plus de trente pays, où elle met en œuvre projets de terrain et expertises. La formation et l'insertion professionnelles sont l'une de ses thématiques d'intervention. 
importante aux ONG comme partenaires privilégiés des bailleurs multilatéraux et bilatéraux. Cette situation de partenariat est facilitée par le caractère non marchand des ONG. À cette époque, certaines ONG pilotent directement des projets de leur agence nationale de coopération (américaine ou canadienne par exemple) et agissent comme intermédiaires entre États et bailleurs.

Dans le même mouvement, les bailleurs encouragent les États à mettre en place des réseaux d'ONG partenaires susceptibles de porter plus efficacement des activités décentralisées, des modes de gestion plus directs des activités d'offre d'enseignement formel et non formel. Les ONG se développent dans les secteurs négligés par les États - prise en charge des adultes, des déscolarisés, alphabétisation, modes d'éducation et de formation non formels -, dans une spécialisation de subsidiarité qui est encore la leur dans les pays du Nord. Dans ce mouvement de « privatisation » des activités éducatives, lié souvent à la défaillance des États, des ONG représentent la société civile, qui parfois prend directement en charge la construction de salles de classe, le recrutement et la rémunération des enseignants. Un large mouvement de décentralisation de la gestion des établissements scolaires promeut, via des ONG, une société civile prenant part aux décisions courantes de gestion et d'administration des écoles. Les ONG se développent dans l'enseignement professionnel, qui se construit d'abord en rapprochant des entreprises (souvent très petites et décentralisées) et de futurs apprenants, puis en mettant en place des solutions souples de repérage des besoins, de formation (des formateurs et de jeunes bénéficiaires), de financement.

Le Forum de Dakar ${ }^{3}$ a initié un réel mouvement pour le développement de la scolarisation primaire universelle et ouvert la voie aux Objectifs du millénaire pour le développement (OMD). Il a conduit, de façon très visible, à un changement profond des termes et des pratiques en matière d'aide. L'engagement de la communauté internationale à financer tout "plan éducatif crédible» a remis les États au centre de la politique éducative et favorisé une stratégie de renforcement des capacités publiques d'intervention dans le domaine qui rompt avec la stratégie passée d'extériorisation et de privatisation des activités financées dans les pays par l'aide internationale. Le développement de l'aide budgétaire ${ }^{4}$ et la disparition progressive de la politique de projets s'inscrivent dans cette perspective, réduisant sur ce dernier plan l'activité directe des ONG. Dans le même temps, le souci de rendre l'aide plus efficace conduit à un développement des activités d'harmonisation de l'aide et d'alignement sur les procédures nationales. Ce mouvement d'entente des bailleurs aux niveaux national et international ainsi que le développement d'un débat mondial sur les enjeux et principes liés à la définition de plans de long terme pour les systèmes éducatifs nationaux ont favorisé le développement de coordinations et de groupements d'ONG, qui portent leur vision au sein des grandes réunions internationales sur l'Éducation pour tous (EPT) et le développement des systèmes éducatifs.

3. Forum de Dakar, voir sur le site de l'Unesco : http://www.unesco.org/education/efa/fr/wef_200o/index.shtml/. 4. L'aide budgétaire peut être sectorielle, lorsqu'elle est inscrite en recette spécifique du département ministériel qu'elle est destinée soutenir, ou globale lorsqu'elle constitue une recette budgétaire non spécifiquement affectée. 
Ces évolutions ont favorisé une mutation des ONG. Si leur rôle de simples relais privés des politiques d'aide s'est affaibli, sauf dans les États en crise (dits États fragiles), où au contraire il s'est présenté comme un mode de fonctionnement temporaire acceptable d'actions de soutien aux populations, le rôle d'interlocuteur des ONG s'est affirmé dans le cadre d'une représentation des populations et des États eux-mêmes dans les rencontres internationales, où s'élaborent les politiques de promotion de l'éducation et du développement durable. Cette position importante des ONG devrait encore se développer si l'on considère les défis auxquels font face les systèmes éducatifs dans les pays en voie de développement et les atouts des ONG pour aider à les relever.

Les progrès accomplis depuis 2000 dans nombre de ces pays ont surtout concerné l'accès à l'éducation, alors que la rétention à l'école est demeurée problématique. De fait, la réduction des inégalités a été très partielle, contrecarrée par le maintien d'évidentes inégalités de carrière scolaire. La qualité des apprentissages et la pertinence nationale de leur contenu ont souvent été négligées, alors qu'elles étaient déjà discutables dans nombre de pays. À ces difficultés caractérisant l'éducation primaire s'ajoutent aujourd'hui celles qu'on peut lier plus directement à l'amélioration incontestable de l'accès à ce niveau d'enseignement et qui posent avec force la question du développement d'un enseignement professionnel pertinent notamment au niveau charnière du post-primaire.

Face aux enjeux des systèmes éducatifs dans les pays en développement, les ONG se sont ainsi progressivement imposées comme des acteurs à part entière de la coopération et de l'action éducative. Si les défis évoqués ci-dessus ont de réelles dimensions techniques et pédagogiques, ils ont évidemment, dans le même temps, une importante dimension financière, alors même que la forte mobilisation budgétaire des États n'a pas toujours trouvé l'équivalent attendu de la communauté internationale. Ces défis auxquels se trouvent confrontés les pays en développement font porter sur les ONG trois types d'attentes au plan de leur contribution au développement de politiques et de systèmes éducatifs :

- innover pour pouvoir ensuite " passer le relais » et faire évoluer les systèmes éducatifs vers des pratiques efficaces et durables;

- agir au plus près des populations, en renforçant les capacités de la société civile ;

- identifier des financements auxquels les États du Sud n'ont pas accès.

Ce dossier ne prétend pas traiter de façon exhaustive la complexité d'un tel sujet. Illustrant des cas, des géographies et des contextes variés, principalement au Sud, les textes reflètent des questionnements et des points de vue de bailleurs, d'ONG, ainsi que de chercheurs ${ }^{5}$. Ils illustrent des positions et des opinions qui ouvrent des débats. Certaines de ces réflexions sont encore naissantes et gagneront à devenir des sujets dont la recherche s'emparera. 


\section{LES APPORTS EN MATIÈRE D'INNOVATION ÉDUCATIVE}

L'innovation éducative cherche à combler des lacunes identifiées du système éducatif, grâce à une proximité avec les acteurs locaux et à la grande liberté de fonctionnement dont jouissent les ONG. Cette innovation peut concerner : la conception et la mise en œuvre de pédagogies spécifiques (pour les élèves ou pour la formation de formateurs); le ciblage de publics particuliers; l'adaptation des réponses aux besoins du marché du travail ; la préparation des jeunes à l'entrée dans la vie active ; la reconnaissance de modes non formels d'éducation ou de formation ; les modalités de gestion, de suivi, de pilotage d'actions, comme la mobilisation du secteur privé et la concertation ; l'accompagnement des processus de décentralisation, de délégation de certaines responsabilités (comme le passage d'examens) à des associations professionnelles, la gestion concertée des écoles par les parents d'élèves, etc.

Les ONG complètent utilement l'action de l'État en tant qu'acteurs directs chargés de la gestion de programmes éducatifs, qui réclament précisément la souplesse d'intervention et de fonctionnement dont elles sont capables. Ces actions « de complémentarité » peuvent être encadrées par un partenariat explicite avec l'État, les bailleurs ou les communautés ou se constituer sur la base d'un " marché d'opérateurs » ouvert à une certaine concurrence entre les organisations intervenantes. Lorsque la formation considérée met en jeu les entreprises et/ou le fonctionnement du marché du travail, comme dans le cas de la formation professionnelle, les structures publiques sont souvent inadaptées aux besoins et les ONG deviennent alors des acteurs quasiment incontournables, leur statut d'organisation à but non lucratif facilitant en outre leurs activités d'intermédiation entre employeurs, employés, personnes formées et financeurs.

On peut ainsi s'attendre au développement du rôle, traditionnel pour les ONG, de promotion de contenus éducatifs plus proches des populations et des États, recourant davantage aux langues nationales, centrés sur les besoins locaux et facilitant l'adoption de rythmes et de calendriers scolaires plus compatibles avec les contraintes des familles.

L'action de l'ONG Sipar ${ }^{6}$ au Cambodge, présentée par Nathalie Dupont et Sothik Hok, illustre l'impact que l'action d'une ONG peut avoir sur une politique nationale. Un module de formation des enseignants sur les «bibliothèques et livres pour enfants » a été conçu par une équipe mixte en réponse à une demande du ministère cambodgien de l'éducation et intégré à la formation initiale des élèves instituteurs du pays. Désormais, l'État cambodgien reconnaît et valorise financièrement ses instituteurs formés comme bibliothécaires.

Il y a un large consensus entre les différents types d'acteurs (comme la table ronde de ce dossier le confirme) sur le fait que les ONG devraient jouer un rôle particulièrement actif dans le développement et la reconnaissance des

\footnotetext{
6. Sipar : soutien à l'initiative privée pour l'aide à la reconstruction.
} 
enseignements non formels, qui sont par nature moins normalisés que l'enseignement de base et permettent la mise en ouvre de parcours plus spécifiques dans les bassins d'emploi, les régions, les pays. Le succès du développement attendu de l'enseignement professionnel dépendra largement de l'investissement dans ces activités d'ONG capables d'aider des entreprises et des marchés informels à prendre leur part de la formation des jeunes, de la régulation de la demande d'éducation et de l'évolution vers des modes d'organisation économiques plus productifs.

L'initiative iLEAD (Initiative for Livehood Education and Development) $\mathrm{d}^{\prime} \mathrm{AEAI}^{7}$ en Inde, présentée par PB Sajeev et Graciela Padoani, est une autre illustration de la capacité d'innovation des ONG selon les besoins identifiés localement. Le programme vise à améliorer le processus d'insertion des jeunes femmes en renforçant leur autonomie sociale, financière et professionnelle. Initiée en 2005, l'action couvre aujourd'hui dix-neuf États en Inde et a permis de former cent mille jeunes, $75 \%$ d'entre eux ayant ensuite obtenu un emploi rémunérateur. AEAI échange régulièrement avec le gouvernement central et appuie différents gouvernements de cette République fédérale en matière de stratégie de formation professionnelle ou de création d'emplois.

La réflexion sur l'alphabétisation au Maroc présentée par Sophie Cerbelle et Mohammed Bougroum questionne pour sa part l'efficacité comparée de l'action des ONG et d'autres acteurs, privés et publics, dans la délivrance de services d'alphabétisation. Les auteurs constatent que l'offre d'alphabétisation des ONG est à la fois importante (48 \% des effectifs du pays en 2010-2011), moins coûteuse du fait de salaires moins élevés et du bénévolat, mais aussi moins efficace, du fait de la précarité des formateurs et de leur niveau de formation plus faible. Si le manque de données sur les coûts ne permet pas une analyse comparée de l'efficience des interventions, un tel constat laisse entrevoir la possibilité pour le gouvernement, au prix d'une stabilisation du statut et des conditions d'exercice des formateurs des ONG, de développer via ces opérateurs une capacité d'accueil à la fois large et relativement peu coûteuse.

La principale difficulté et le principal enjeu, pour les ONG, sont peutêtre de pérenniser les actions et de poser les bases de leur changement d'échelle. Les deux articles présentant des actions d'ONG sont révélateurs du fait qu'il n'existe pas de "modèle type » : dans le cas du Sipar, il s'agit d'une intégration à une politique nationale; dans celui d'AEAI, du développement d'un modèle par adaptation/diffusion géographique progressive. L'exemple du Sipar illustre aussi les étapes charnières d'évolution d'une ONG étrangère au pays d'intervention : suite à la fin d'un financement, la structure a dû muter en ONG locale, afin de poursuivre ses actions dans le pays. S'ajoute à cela une difficulté générique supplémentaire, plus organisationnelle : celle de concilier financements de court terme sur base de projets et actions de long terme.

7. Aide et Action International. 


\section{Dialogue, PLAidoyer ET CONTRIBUTION AUX POLITIQUES PUBLIQUES}

Le dialogue avec la société civile et le renforcement des capacités d'action de cette dernière représentent un apport majeur de l'action des ONG, qui est largement reconnu comme tel par les bailleurs, les États et les populations.

Le plaidoyer, que ce soit au niveau local, national, régional ou international, constitue pour les ONG un complément et/ou une alternative à l'activité opérationnelle conduite, pour faire évoluer les politiques et les pratiques. Il est également intéressant d'examiner comment, et avec quel succès, les ONG ont investi en plus grand nombre le champ du plaidoyer en réponse notamment à l'harmonisation et à l'intégration des actions des bailleurs.

Philippe Ryfman propose une analyse de la relative sous-représentation du champ "éducation " au sein des ONG françaises, alors que l'éducation a historiquement constitué un axe fort de l'aide publique au développement (APD) et des politiques publiques, notamment dans le cadre des Objectifs du millénaire pour le développement (OMD). Il présente l'évolution du paysage des ONG, de l'espace public et des financements non gouvernementaux. Il livre aussi, de manière inédite, des éléments d'analyse des contraintes organisationnelles et des priorités des grandes et moyennes $\mathrm{ONG}$, qui les ont conduites à délaisser l'éducation, malgré des mandats qui vont s'élargissant depuis une quinzaine d'années, dans un triple souci de sécurisation financière, de renforcement de la structure et d'optimisation de la gestion. Mais le plaidoyer en matière d'éducation est difficile à porter, car le thème "éducation pour tous ", consensuel, "en affadit probablement la portée et est moins facilement articulable avec d'autres causes au sein desquelles il tend à se diluer ». Par ailleurs, le grand public y est moins sensible qu'à d'autres sujets. Enfin, la recherche de financements se heurte, outre à la crise actuelle, au « retour de l'État comme acteur principal du développement au Sud sur les axes considérés comme relevant fondamentalement du régalien ».

Selon les experts invités à participer à une table ronde organisée pour ce dossier ${ }^{8}$, les ONG contribuent à écouter, à mobiliser et à épauler les acteurs de l'action éducative, en intervenant à un niveau local, dans la perspective d'une formation plus équitable et efficace. Une des principales limites de leur intervention est la difficulté à faire en sorte que leurs actions soient reprises et généralisées dans le cadre d'une politique publique, pour les inscrire de façon

8. La table ronde, animée par Sandra Barlet (Gret), a réuni le 30 septembre 2011 : Roland Biache (Solidarité laïque), Valérie Huguenin, Jacques Marchand et Ghislain Kouton (AFD), Bernard Dumont, André Gauron, et enfin Gilbert Migan (ministère en charge de la formation technique et professionnelle du Bénin). 
pérenne dans l'évolution du système éducatif. Si le dialogue avec la société civile est ainsi essentiel, le plaidoyer pour l'appropriation par l'institution des messages et des actions est tout aussi capital.

Un autre point fondamental est évoqué dans cette table ronde: la concertation entre ONG, et plus largement entre acteurs de l'éducation - bailleurs compris -, est un élément majeur pour la cohérence d'ensemble des interventions. Au Cambodge par exemple, une plateforme des 85 ONG actives dans le champ de l'éducation permet aux ONG de se concerter et de réguler leur dialogue avec l'État, qui coordonne leur action dans son ensemble. L'enjeu de la concertation rejoint d'ailleurs la question de la forte concurrence qui existe entre elles, abordée également par Philippe Ryfman, qui montre la nécessité pour les ONG de sécuriser leurs ressources dans un contexte de stabilité de la donation privée, de contribution marginale de l'aide publique au développement transitant par les ONG et d'émergence de financeurs alternatifs.

Dans le cas particulier des États fragiles, on peut, comme le fait Thomas Poirier, interroger les limites de l'apport de la société civile, ce qui l'amène à une autre interrogation sur le positionnement des ONG dans ces contextes spécifiques. Soulignant la nécessité de restaurer une puissance publique forte, l'auteur estime que renforcer la capacité de la société civile à gérer localement certains services ne va pas de soi, et qu'une gestion locale de l'éducation peut entraîner un risque d'abandon par l'État de sa responsabilité en matière d'éducation. Ce qui est en jeu ici est la conception du rôle de l'État. André Gauron rappelle, dans la table ronde, que la coopération institutionnelle française a plutôt une tradition d'appui aux administrations en charge de l'éducation (bien qu'elle ait évolué depuis plusieurs années vers une mobilisation de plus en plus forte des acteurs locaux), et que d'autres coopérations, comme celles des pays anglo-saxons, ciblent certains publics (par exemple, les déscolarisés du primaire) et réservent à l'État une fonction de définition du cadre des contenus et des certifications. Cela ouvre, selon nous, un large champ à l'action de la société civile sans pour autant priver l'État de ses prérogatives. Deux approches s'opposent donc: celle de la restauration d'une puissance publique forte comme voie privilégiée du développement des États fragiles et celle qui souligne au contraire l'intérêt de l'apport spécifique des ONG y compris dans ces États, pour promouvoir une éducation décentralisée. Comme l'indique T. Poirier, il convient de toute façon de relativiser l'agenda international et les objectifs pour ces pays, qu'ils «ne seront pas à même de tenir compte tenu de leurs difficultés et de leur fragilité ».

\section{LES NOUVEAUX FINANCEMENTS}

On peut s'attendre à ce que des ONG contribuent à la mobilisation de nouvelles ressources financières, d'abord en rappelant aux États développés et aux bailleurs leurs engagements partiellement tenus, ensuite en aidant à la recherche de modes de financement innovants: entreprises, fondations, 
mécénats, nouveaux bailleurs, lignes de financements auprès des bailleurs bi- ou multilatéraux, non accessibles directement aux États, ou encore développement de partenariats public-privé, à l'exemple de cursus montés en collaboration avec des professionnels et les organisations les représentant. Philippe Ryfman relève que les stratégies actuelles d'extension de mandat des ONG les poussent à se tourner vers des financements alternatifs, et notamment vers les fondations philanthropiques et les nouveaux pays donateurs. Les ONG peuvent également intervenir pour combler les manques de l'action globale de l'État dans des marges qui sont fonction des arbitrages publics et des moyens disponibles. Plus que de la complémentarité (organisée) évoquée précédemment, il s'agit ici de se substituer temporairement aux activités de l'État auquel ses moyens, ses choix implicites et sa gestion des fonds publics ne permettent pas de délivrer en tout lieu et à tous les groupes les services collectifs qu'il est censé offrir.

Si le Gret a mené en 2008 une étude portant sur l'action des ONG françaises ${ }^{9}$ au Sud en matière d'éducation, qui comprenait une photographie des sources de financement des ONG enquêtées (avec des financements privés légèrement prépondérants), l'identification et la gestion de financements alternatifs à ceux qui alimentent le système éducatif formel via les gouvernements n'avait pas encore, à notre connaissance, fait l'objet de recherches approfondies à un niveau international.

Stephen Ball et Antonio Olmedo présentent ici quelques éléments d'une recherche en cours, à paraître en 2012, sur l'arrivée de ces nouveaux acteurs dans le secteur du développement international que sont les "nouveaux philanthropes" du "don à but "lucratif" ». Ces derniers voient ces pratiques axées sur le marché comme "des moyens plus efficaces de favoriser l'accès à l'éducation à un plus grand nombre et d'améliorer la qualité de l'éducation que ne le seraient les gouvernements ou l'aide traditionnelle ou encore le secteur caritatif ». Par exemple, au cours de la décennie passée, quatre fondations philanthropiques ont alloué au total 4,4 milliards de dollars à différents programmes liés à la réforme scolaire aux États-Unis. En Afrique, une organisation se fixe pour but d'atteindre un million d'enfants au cours des trois prochaines années et en attend des retours sur investissements. Les auteurs soulignent que "les processus en jeu ici agissent à la fois "sur" et "contre" l'État et le secteur public de l'éducation ». À travers leur action philanthropique et leurs organisations non lucratives (fondations, organismes caritatifs, ONG, etc.), ces nouveaux philanthropes seraient à même d'influencer, voire de définir les priorités publiques en matière d'éducation.

Pour ces deux auteurs cette nouvelle conception de la philanthropie «brouille à dessein la frontière entre le monde des affaires, le monde de l'entreprise, le domaine du développement et celui du bien public». Il resterait à 
mettre en perspective l'action des "philantrepreneurs " avec les autres sources de financement dans le domaine de l'éducation et à analyser plus loin les interactions de ces acteurs avec les politiques et systèmes éducatifs. De même, la question de la recherche de bénéfices par ces « nouveaux philanthropes » ouvre un débat qui devrait accompagner nécessairement le mouvement actuel pour la recherche de (nouvelles) ressources pour la promotion de l'éducation dans le monde mais qui concerne sans doute tout autant les financeurs traditionnels de l'aide et même les ONG. On remarquera d'ailleurs que l'interrogation sur l'efficacité de l'aide est aujourd'hui partagée par la plupart des intervenants dans le domaine du développement et qu'elle s'exprime aussi par des termes qui empruntent à la gestion et aux pratiques commerciales.

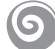

Nous constatons, à la lecture de ces différents textes, que la question " ONG et éducation », est à la fois plurielle, mouvante et complexe. Plurielle, car les structures concernées - des associations de solidarité internationales aux associations locales - sont extrêmement variées en termes de positionnement, de type d'activité, de conviction idéologique, de sources de financements, de mode d'intervention, d'organisation. En particulier, tenter une analyse conjointe et globale concernant à la fois les ONG locales et les associations de solidarité internationales est un exercice périlleux - on note d'ailleurs que, le plus souvent, les auteurs ont analysé un seul type d'intervenant. Mouvante, car ces structures évoluent vite, sujettes à des contextes d'intervention instables, à des financements contraints, à une exigence de taille critique d'intervention pour survivre et à l'apparition de nouveaux acteurs, opérateurs et bailleurs. Complexe enfin, car, en matière de développement, comme le montrent les différents points de vue qui s'expriment dans ce dossier, il n'y a pas de vérité, même s'il existe des consensus qu'il est toujours bon de questionner. Cette complexité tient également au fait que l'éducation est au cœur de la question du développement de la force vive des pays concernés et qu'elle est probablement aujourd'hui leur enjeu le plus prioritaire et le plus urgent, pour que progresse demain leur capital intellectuel et économique.

Nous pensions, en entamant cette réflexion à l'invitation de la Revue internationale d'éducation de Sèvres, qu'il serait difficile de fournir une vue globale du rôle des ONG dans le domaine de l'éducation dans les pays en développement. La sérieuse étude bibliographique établie par Hélène Beaucher en fin de dossier montre qu'il est possible de trouver des analyses de cas précis, mais que les recherches globales sur le sujet sont partielles voire inexistantes. Nous espérons que ce dossier permet d'introduire cette question et ouvrira des débats stimulants. 